\title{
Fatores associados ao uso não urgente de unidades de pronto atendimento: uma abordagem multinível
}

\section{Factors associated withnon-emergency use of Emergency Care Units: a multilevel approach}

Lidiane Cintia de Souza', Glaucia Maria Bovi Ambrosano², Katarinne Lima Moraes³, Emílio Prado da Fonseca' (DD, Fábio Luiz Mialhe ${ }^{4}$

\author{
- Programa de Pós-graduação em Odontologia, Área de concentração Saúde Coletiva, Faculdade de Odontologia de Piracicaba \\ Universidade Estadual de Campinas (UNICAMP) - Piracicaba (SP), Brasil. \\ ${ }_{2}^{2}$ Departamento de Ciências da Saúde e Odontologia Infantil, Área de Bioestatística, Faculdade de Odontologia de Piracicaba, \\ Universidade Estadual de Campinas (UNICAMP) - Piracicaba (SP), Brasil. \\ ${ }^{3}$ Curso de Enfermagem, Universidade Federal de Jataí (UFJ) - Jataí (GO), Brasil. \\ ${ }^{4}$ Departamento de Ciências da Saúde e Odontologia Infantil, Área de Educação e Promoção da Saúde, Faculdade de \\ Odontologia de Piracicaba, Universidade Estadual de Campinas (UNICAMP) - Piracicaba (SP), Brasil.
}

Como citar: Souza LC, Ambrosano GMB, Moraes KL, Fonseca EP, Mialhe FL. Fatores associados ao uso não urgente de unidades de pronto atendimento: uma abordagem multinível. Cad Saúde Colet, 2020;28(1):56-65.

https://doi.org/10.1590/1414-462X202000280354

\section{Resumo}

Introdução: estudos no Brasil e em diversos países indicam que os serviços de urgência são frequentemente utilizados como portas de entrada para os sistemas de saúde, sobrecarregando-os e impactando na qualidade do serviço prestado à população. Entretanto, pouco se conhece sobre esse fenômeno nas unidades de pronto atendimento (UPAs) do nosso país. Objetivo: investigar as variáveis associadas ao uso inadequado de duas UPAs em um município de grande porte. Método: estudo transversal com uma amostra de 756 indivíduos que analisou as variáveis individuais, contextuais e dos serviços associadas ao uso inapropriado de UPAs por meio de um modelo de regressão logística múltipla e hierarquizada. Resultados: o modelo final de regressão demonstrou que os indivíduos que apresentavam autopercepção de que sua condição era um caso de emergência e cuja idade era igual a ou acima de 60 anos apresentaram mais chances de procurarem esses serviços por motivos não urgentes. Conclusão: variáveis individuais estiveram associadas ao uso não urgente dos serviços das UPAs. Estudos futuros de cunho qualitativo poderão auxiliar a compreender os motivos do uso inadequado desses serviços por tais indivíduos.

Palavras-chave: epidemiologia; serviços médicos de emergência; necessidades e demandas de serviços de saúde; serviços de informação.

\begin{abstract}
Background: Studies in Brazil and in several countries indicate that emergency services are often used as gateways to health systems, overburdening them and impacting on the quality of service provided to the population. However, little is known about this phenomenon in the Emergency Care Units (EUCs) of our country. Objective: To investigate the variables associated with the inadequate use of two EUCs in a large municipality. Method: A cross-sectional study was carried out with a sample of 756 individuals that analyzed individual, contextual and service-related variables associated with the inappropriate use of EUCs through a hierarchical multiple logistic regression model. Results: The final regression model showed that individuals who had self-perceived their condition as an emergency and whose age was equal to or older than 60 years presented high odds to seek these services for non-urgent reasons. Conclusions: Individual
\end{abstract}

Trabalho realizado na Universidade Estadual de Campinas (UNICAMP) - Piracicaba (SP), Brasil.

Correspondência: Fábio Luiz Mialhe. E-mail: mialhe@unicamp.br

Fonte de financiamento: nenhuma.

Conflito de interesses: nada a declarar.

Recebido em: Dez. 28, 2017. Aprovado em: Maio 14, 2019 cadernos

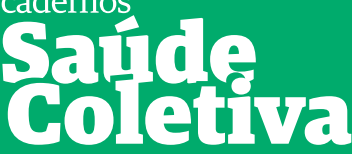

ISSN 2358-291X (Online)

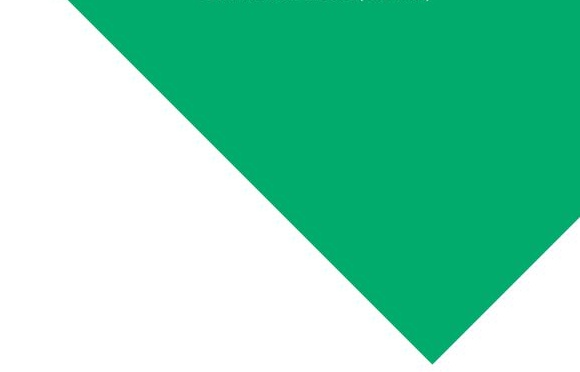

\section{.}


variables were associated with non-urgent use of the ECUs. Future studies of a qualitative nature may help to understand the reasons for the inadequate use of these services by these individuals.

Keywords: epidemiology; emergency medical services; needs and demand health services; information services.

\section{INTRODUÇÃO}

A reforma da Política Nacional de Atenção às Urgências (Portaria nº 1.600, de 7 de julho de 2011) adotou como estratégia nuclear a implantação da rede temática prioritária de atenção às urgências e emergências (RUE) com vistas a assegurar ao usuário o conjunto de ações e serviços em situações de urgência e emergência com resolutividade e em tempo oportuno'. Entre os componentes dessa rede, estão as unidades de pronto atendimento (UPAs) que funcionam como unidades intermediárias entre a atenção básica e os hospitais e atuam em conformidade com a lógica de acolhimento e de classificação de risco, auxiliando no alívio da demanda aos hospitais e prontos-socorros pertencentes ao Sistema Único de Saúde (SUS) ${ }^{1}$.

As transições epidemiológica e demográfica vivenciados no Brasil indicam uma acelerada progressão de mortes por causas externas e das doenças cardiovasculares, o que demanda uma nova conformação do sistema de saúde brasileiro ${ }^{2}$. Nesse sentido, o enfrentamento das situações de urgência e emergência e de suas causas requer não apenas assistência imediata, mas inclui ações de promoção da saúde e prevenção de doenças e agravos, com tratamento contínuo e eficaz das doenças crônicas, reabilitação e cuidados paliativos ${ }^{1}$. Ademais, o atendimento às urgências deve estar amparado efetivamente em todos os níveis de atenção, cada uma dentro das suas limitações de resolutividade, inclusive na atenção básica ${ }^{3}$.

No Brasil, as unidades de urgência e emergência, historicamente, são consideradas a referência para o tratamento de problemas de saúde pela população, por serem ambientes com atendimento rápido e resolutivo ${ }^{4}$. Assim sendo, há uma compreensão equivocada da finalidade do serviço de urgência e emergência, que é vista como alternativa para a falta de resolutividade da atenção básica.

Estudos apontam a existência de demandas desnecessárias em serviços de urgência e que estão relacionadas a fatores sociodemográficos, culturais, do acesso aos serviços e à organização do sistema de saúde 5 . A utilização inapropriada dos serviços de urgência como porta de entrada para o sistema de saúde pode sobrecarregar o serviço (superlotação) e, consequentemente, impactar a qualidade dos serviços prestados nesses serviços ${ }^{5,6}$. Ademais, tal fato pode aumentar os custos desnecessários para o sistema de saúde e induzir uma forma de assistência que não produz vínculo com os usuários dos serviços, pois os indivíduos recebem apenas o tratamento dos sintomas agudos ${ }^{7}$. Entretanto, a produção científica nacional sobre quais fatores levam o uso inapropriado dos serviços de urgência e emergência nas UPAs ainda é escassa, concentrando-se de forma substancial na região Sul do país e em contextos de serviços de urgências hospitalares ${ }^{4,8,9}$.

Com base no exposto, o objetivo deste estudo foi investigar as variáveis individuais, contextuais e dos serviços de saúde associadas à utilização inapropriada de duas UPAs localizadas em um município de grande porte do estado de São Paulo.

\section{MÉTODO}

Trata-se de um estudo transversal realizado no município de Piracicaba (SP), distante 165 km da capital, São Paulo, e que em 2012 contava com uma população estimada em 364.571 habitantes e índice de desenvolvimento humano (IDH) de $0,785^{10}$.

\section{Seleção das UPAs}

Fizeram parte do estudo duas das quatro UPAs do município e que haviam implantado o sistema de acolhimento com avaliação e classificação de risco baseado no Sistema de Triagem Manchester, o qual identifica o estado de saúde do indivíduo em quatro níveis de gravidade, 
por meio de cores: vermelho (emergente); amarelo (urgente); verde (pouco urgente); azul (não urgente) ${ }^{11}$.

A amostra foi dimensionada para detectar odds ratio acima de 1,50, para taxa de exposto/não exposto na variável independente de 1:1 a 1:5, nível de confiança de $95 \%$ e poder do teste de, no mínimo, $80 \%$ para todas as associações analisadas, resultando em 756 voluntários.

\section{Seleção dos indivíduos}

A população do estudo foi constituída por usuários maiores de 18 anos de idade, de ambos os sexos, que procuraram as duas UPAs, no período de julho a dezembro de 2012, e que, na recepção, haviam sido classificadas de acordo com o critério de risco no processo de acolhimento ${ }^{11}$. Após terem passado pela classificação de risco e consulta médica, os usuários foram convidados pela pesquisadora a participarem da pesquisa e assinaram um termo de consentimento. No caso dos usuários classificados como urgentes, foi realizada entrevista com aqueles que apresentavam condições de responder às questões e foram excluídos do estudo indivíduos que não residiam em Piracicaba.

Foi elaborado um questionário com base nos estudos anteriores que investigaram o mesmo tema, caracterizando as variáveis independentes do estudo ${ }^{9}$. A variável dependente caracterizou-se pelas classificações das UPAs em urgentes ou não urgentes. Os questionários foram aplicados por uma entrevistadora previamente treinada para o estudo, que permanecia nas UPAs entre as $8 \mathrm{~h}$ e as $18 \mathrm{~h}$, de segunda à sexta-feira. Coletas das informações foram realizadas na sala de espera das duas UPAs e previamente ao atendimento. Selecionou-se esse período, pois, segundo um estudo de Gomide et al. ${ }^{12}$, há mais estabilidade na procura por serviços de urgência em dias úteis e períodos diurnos (manhã e tarde), já que nesses dias e horários as UBSs e USFs também estão abertas para atendimento (distorção no sistema) ${ }^{12}$.

Um estudo piloto foi realizado com 20 usuários para verificar a clareza e o entendimento das perguntas, o padrão de respostas e testar o método de entrevista.

\section{Variáveis do estudo}

Foram obtidas dos sujeitos informações sobre quatro grupos de variáveis: 1. Variáveis sociodemográficas: idade ( 18 a 44, 45 a 59, 60 ou mais), sexo, cor da pele (branco/não branco) e situação conjugal atual (com parceiro/sem parceiro); 2. Variáveis socioeconômicas: renda familiar, escolaridade, tempo de deslocamento até a UPA e se possui plano de saúde (sim, não); 3. Variáveis relacionadas ao uso dos serviços: o atendimento da UBS/USF do bairro é (muito bom/bom, regular/ruim, nunca foi), quantas vezes procurou a UPA e a USF/UBS nos últimos seis meses (até três vezes; mais de três vezes), dificuldade de acesso à atenção básica (sim não), já veio antes à UPA (sim, não); 4. Autorrelato de necessidades em saúde: estado de saúde (muito bom/bom, regular, ruim), doença crônica (sim, não), percepção se seu estado de saúde é urgente (sim, não), duração dos sintomas (um a três dias $\leq 4$ a 30 dias, $>30$ dias). Todas foram selecionadas com base em estudos prévios que verificaram associações com a variável desfecho $0^{4-9}$.

\section{Análise dos dados}

As associações entre a classificação do usuário como urgente ou não urgente e as variáveis independentes foram analisadas por meio de modelo de regressão logística múltipla e hierarquizado de três níveis pelo procedimento PROC GENMOD do programa SAS.

Para construir a hierarquização do modelo, utilizou-se o referencial teórico de Andersen (1995), que caracterizou o acesso aos serviços de saúde segundo grupos de variáveis, que abrangem da entrada no serviço de saúde até o cuidado prestado ${ }^{13}$. Nesse sentido, o acesso é compreendido como produto da relação do indivíduo/usuário (demanda) com as características dos serviços (oferta) para explicar a existência ou não de utilização de serviços de saúde ${ }^{13}$. O autor desenvolveu um modelo para análise do acesso embasado no trinômio disponibilidade, 
acessibilidade e aceitabilidade (necessidade percebida) e que permite estabelecer a relação oferta/demanda, o volume e o tipo de serviços existentes, a capacidade financeira (affordability), a organização da rede assistencial e tomando por base as características da população ${ }^{13}$. Mais do que aspectos mensuráveis e que permitem uma avaliação externa, o acesso é determinado por questões políticas, assistencial/operacional de oferta de serviços de saúde e individuais (objetivas e subjetivas) $^{13}$. A Figura 1 apresenta uma representação das variáveis que fizeram parte de cada nível, sendo os fatores de predisposição: idade, sexo, cor da pele, situação conjugal, escolaridade e renda; fatores facilitadores: tempo de deslocamento até a UPA, possui plano de saúde, qualidade no atendimento da UBS/PSF, quantas vezes visitou a unidade de saúde nos últimos seis meses, dificuldade de acesso na atenção básica; necessidades percebidas: autoavaliação de saúde, portador de doença crônica, percepção de emergência e tempo de surgimento dos primeiros sintomas.

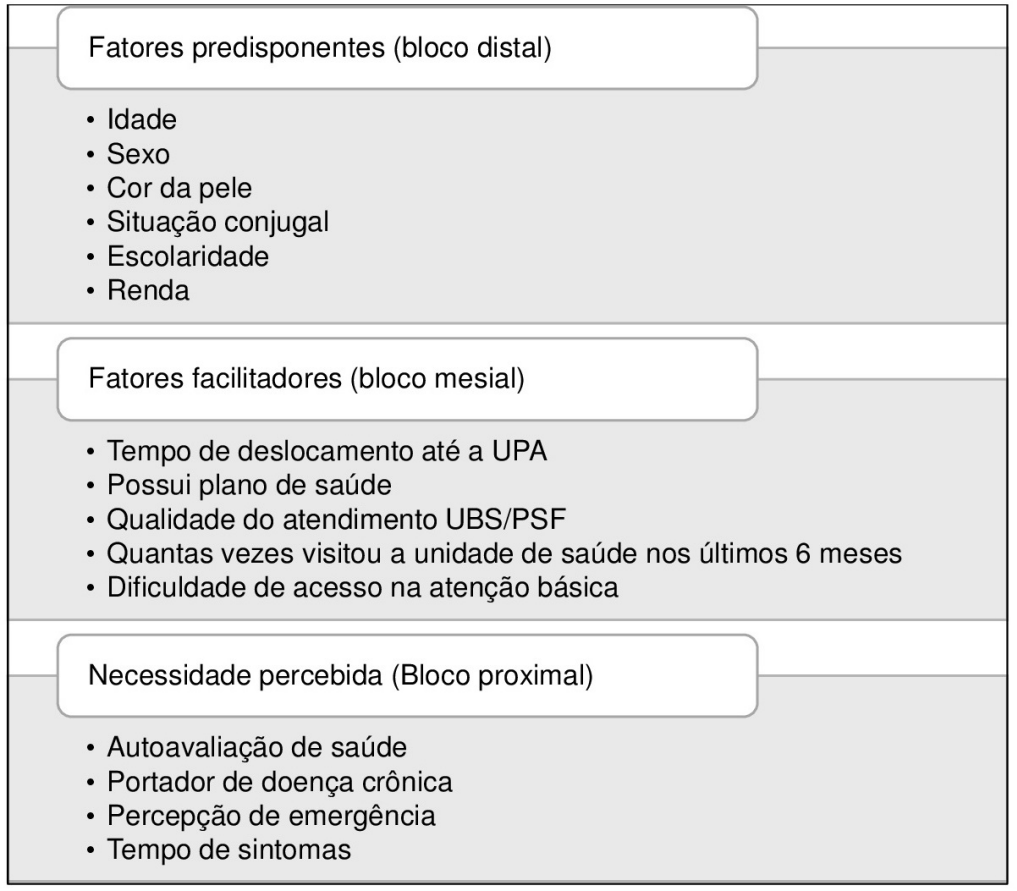

Figura 1. Modelo de análise multivariado e hierarquizado de acesso a serviços de urgência na modalidade UPA - Piracicaba, 2016.

Foram testadas no modelo de regressão logística múltipla as variáveis com $p \leq 0,20$ em cada nível, permanecendo no modelo aquelas que continuaram associadas à classificação da urgência ou não com $p \leq 0,05$ após o ajuste para as variáveis do mesmo nível e para os níveis subsequentes. A significância estatística referente à introdução de cada variável no modelo foi analisada pelo $\mathrm{p}$-valor e o ajuste do modelo como um todo foi avaliado pela estatística - 2 vezes o logaritmo da verossimilhança. Todas as análises foram realizadas no programa estatístico SAS versão 9.2. A Figura 1 apresenta o modelo de análise multivariado e hierarquizado de acesso a serviços de urgência na modalidade UPA utilizado no presente estudo.

\section{RESULTADOS}

A descrição dos dados e os resultados das análises bivariadas por blocos são apresentados nas Tabelas 1-3. Entre os 756 adultos entrevistados, 374 (49,5\%) possuíam idade entre 18 e 44 anos, 394 (52,1\%) eram do sexo feminino, 461 (61,0\%) eram brancos, $385(50,9 \%)$ não tinham parceiro, $349(46,2 \%)$ apresentavam baixa escolaridade e $460(60,8 \%)$ possuíam renda familiar de até três salários mínimos. Do total dos usuários entrevistados, 347 (45,9\%) foram 
classificados pelas UPAs como não urgentes pelo sistema de acolhimento com avaliação e classificação de risco. Quanto à idade, os usuários não urgentes apresentaram média de $41,24$ anos (DP $\pm 17,23$ ), enquanto os urgentes, 52,69 anos (DP $\pm 19,4)$.

Tabela 1. Resultados das análises individuais da associação das variáveis do bloco distal com o tipo de risco (urgente e não urgente) - Piracicaba-SP, 2016.

\begin{tabular}{|c|c|c|c|c|c|c|c|c|c|}
\hline \multirow{3}{*}{ Variáveis } & \multirow{3}{*}{$\mathbf{N}$} & \multirow{3}{*}{$\%$} & \multicolumn{4}{|c|}{ Urgente } & \multirow{3}{*}{ OR } & \multirow{3}{*}{ IC95\% } & \multirow{3}{*}{ p-valor } \\
\hline & & & \multicolumn{2}{|c|}{ Sim } & \multicolumn{2}{|c|}{ Não } & & & \\
\hline & & & $\mathbf{n}$ & $\%$ & $\mathbf{n}$ & $\%$ & & & \\
\hline \multicolumn{10}{|l|}{ Idade } \\
\hline 18 a 44 anos & 374 & 49.5 & 252 & 67.4 & 122 & 32.6 & 1 & & \\
\hline 45 a 59 anos & 187 & 24.7 & 92 & 49.2 & 95 & 50.8 & 2,13 & $1,49-3,05$ & $<0,0001$ \\
\hline 60 anos ou mais & 195 & 25.8 & 65 & 33.3 & 130 & 66.7 & 4,13 & $2,86-5,97$ & 0,0017 \\
\hline \multicolumn{10}{|l|}{ Sexo } \\
\hline Feminino & 394 & 52.1 & 225 & 57.1 & 169 & 42.9 & 1 & & \\
\hline Masculino & 362 & 47.9 & 184 & 50.8 & 178 & 49.2 & 1,29 & $0,97-1,72$ & 0,0838 \\
\hline \multicolumn{10}{|l|}{ Cor da pele } \\
\hline Branca & 461 & 61.0 & 236 & 51.2 & 225 & 48.8 & 1,35 & $1,01-1,82$ & 0,0452 \\
\hline Não branca & 295 & 39.0 & 173 & 58.6 & 122 & 41.3 & 1 & & \\
\hline \multicolumn{10}{|l|}{ Situação conjugal } \\
\hline Sem parceiro & 385 & 50.9 & 220 & 57.1 & 165 & 42.9 & 1 & & \\
\hline Com parceiro & 371 & 49.1 & 189 & 50.9 & 182 & 49.1 & 1,28 & $0,96-1,71$ & 0,0874 \\
\hline \multicolumn{10}{|l|}{ Escolaridade } \\
\hline $\begin{array}{l}\text { Até fundamental } \\
\text { incompleto }\end{array}$ & 349 & 46.2 & 154 & 44.1 & 195 & 55.9 & 2,29 & $1,68-3,12$ & $<0,0001$ \\
\hline $\begin{array}{l}\text { Fundamental completo } \\
\text { ou médio incompleto }\end{array}$ & 84 & 11.1 & 47 & 56 & 37 & 44.0 & 1,42 & $0,88-2,32$ & 0,1552 \\
\hline $\begin{array}{l}\text { Pelo menos médio } \\
\text { completo }\end{array}$ & 323 & 42.7 & 208 & 64.4 & 115 & 35.6 & 1 & & \\
\hline \multicolumn{10}{|l|}{ Renda } \\
\hline Até $3 \mathrm{SM}$ & 460 & 60.9 & 244 & 53.0 & 216 & 47.0 & 1,12 & $0,83-1,50$ & 0,4678 \\
\hline$\geq 3 \mathrm{SM}$ & 296 & 39.1 & 165 & 55.7 & 131 & 44.3 & 1 & & \\
\hline
\end{tabular}

OR: odds ratio; IC95\%: intervalo de confiança de $95 \%$

Para as variáveis do bloco mesial (Tabela 2), 661 (87,4\%) dos indivíduos atendidos despenderam menos de 30 minutos de deslocamento até a UPA, $666(88,1 \%)$ não possuíam plano de saúde, $332(43,9 \%)$ avaliaram positivamente o atendimento recebido na atenção básica e 576 (76,2\%) consultaram a USF até três vezes nos últimos seis meses. Entretanto, 327 (43,3\%) dos entrevistados relataram dificuldade no acesso à atenção básica. 
Tabela 2. Resultados das análises individuais da associação das variáveis do bloco mesial com o tipo de risco (urgente e não urgente) - Piracicaba-SP, 2016.

\begin{tabular}{|c|c|c|c|c|c|c|c|c|c|}
\hline \multirow{3}{*}{ Variáveis } & \multirow{3}{*}{$\mathbf{N}$} & \multirow{3}{*}{$\%$} & \multicolumn{4}{|c|}{ Urgente } & \multirow{3}{*}{ OR } & \multirow{3}{*}{ IC95\% } & \multirow{3}{*}{ p-valor } \\
\hline & & & \multicolumn{2}{|c|}{ Sim } & \multicolumn{2}{|c|}{ Não } & & & \\
\hline & & & $\mathbf{n}$ & $\%$ & $\mathbf{n}$ & $\%$ & & & \\
\hline \multicolumn{10}{|c|}{$\begin{array}{l}\text { Tempo de deslocamento } \\
\text { até a UPA }\end{array}$} \\
\hline$<15$ minutos & 425 & 56.2 & 211 & 49.6 & 214 & 50.4 & 1,82 & $1,15-2,88$ & 0,0109 \\
\hline De 15 a $30 \mathrm{~min}$ & 236 & 31.2 & 137 & 58.0 & 99 & 42.0 & 1,30 & $0,79-2,12$ & 0,3017 \\
\hline$>30$ minutos & 95 & 12.6 & 61 & 64.2 & 34 & 36.1 & 1 & & \\
\hline \multicolumn{10}{|l|}{ Plano de saúde } \\
\hline Não & 666 & 88.1 & 352 & 52.9 & 314 & 47.1 & 1,54 & $0,98-2,43$ & 0,0625 \\
\hline Sim & 90 & 11.9 & 57 & 63.3 & 33 & 36.7 & 1 & & \\
\hline \multicolumn{10}{|c|}{$\begin{array}{l}\text { Qualidade de } \\
\text { atendimento da UBS/PSF }\end{array}$} \\
\hline Muito bom/bom & 332 & 43.9 & 171 & 51.5 & 161 & 48.5 & 1,07 & $0,69-1,65$ & 0,7572 \\
\hline Regular/ruim & 315 & 41.7 & 180 & 57.1 & 135 & 42.9 & 0,85 & $0,55-1,32$ & 0,4760 \\
\hline Nunca foi & 109 & 14.4 & 58 & 53.2 & 51 & 46.8 & 1 & & \\
\hline \multicolumn{10}{|c|}{$\begin{array}{l}\text { Consultou a USF nos } \\
\text { últimos seis meses }\end{array}$} \\
\hline Até três vezes & 576 & 76.2 & 328 & 56.9 & 248 & 43.1 & 1 & & \\
\hline Mais de três vezes & 180 & 23.8 & 81 & 45 & 99 & 55.0 & 1,62 & $1,15-2,26$ & 0,0052 \\
\hline \multicolumn{10}{|c|}{$\begin{array}{l}\text { Dificuldade de acesso } \\
\text { à atenção básica }\end{array}$} \\
\hline Não & 301 & 39.8 & 146 & 48.5 & 155 & 51.5 & 1,50 & $0,99-2,28$ & 0,0140 \\
\hline Sim & 327 & 43.3 & 188 & 57.5 & 139 & 42.5 & 1,05 & $0,69-1,58$ & 0,3058 \\
\hline Não utilizo & 138 & 16.9 & 75 & 58.6 & 53 & 41.4 & 1 & & \\
\hline
\end{tabular}

OR: odds ratio; IC95\%: intervalo de confiança de $95 \%$

Na Tabela 3 (bloco proximal), verificou-se que 446 (59,0\%) dos indivíduos autoavaliaram seu estado de saúde como satisfatório. Em relação à presença de doença crônica, 379 (50,1\%) dos entrevistados relataram não possuírem. A percepção de necessidade de atendimento de emergência foi positiva para $540(71,4 \%)$ dos entrevistados. O tempo dos sintomas que motivaram a procura pela UPA foi relatado como inferior a três dias para $491(65,2 \%)$ dos entrevistados. 
Tabela 3. Resultados das análises individuais da associação das variáveis do bloco proximal com o risco (urgente e não urgente) - Piracicaba-SP, 2016.

\begin{tabular}{|c|c|c|c|c|c|c|c|c|c|}
\hline \multirow{3}{*}{ Variáveis } & \multirow{3}{*}{$\mathbf{N}$} & \multirow{3}{*}{$\%$} & \multicolumn{4}{|c|}{ Urgente } & \multirow{3}{*}{$\mathbf{O R}^{*}$} & \multirow{3}{*}{ IC95\% } & \multirow{3}{*}{ p-valor } \\
\hline & & & \multicolumn{2}{|c|}{ Sim } & \multicolumn{2}{|c|}{ Não } & & & \\
\hline & & & $\mathbf{n}$ & $\%$ & $\mathbf{n}$ & $\%$ & & & \\
\hline \multicolumn{10}{|c|}{ Autoavaliação de saúde } \\
\hline Muito bom/bom & 446 & 59.0 & 265 & 59.4 & 181 & 40.6 & 1 & & \\
\hline Regular & 223 & 29.5 & 115 & 51.6 & 108 & 48.4 & 1,38 & $0,99-1,90$ & 0,0537 \\
\hline Ruim & 87 & 11.5 & 29 & 33.3 & 58 & 66.7 & 2,93 & $1,80-4,75$ & $<0,0001$ \\
\hline \multicolumn{10}{|l|}{ Doença crônica } \\
\hline Sim & 377 & 49.9 & 170 & 45.1 & 207 & 54,9 & 2,08 & $1,55-2,78$ & $<0,0001$ \\
\hline Não & 379 & 50.1 & 239 & 63.1 & 140 & 36,9 & 1 & & \\
\hline \multicolumn{10}{|l|}{$\begin{array}{l}\text { Percepção da } \\
\text { emergência }\end{array}$} \\
\hline Sim & 540 & 71.4 & 217 & 40.2 & 323 & 59.8 & 11,91 & $7,53-18,82$ & $<0,0001$ \\
\hline Não & 216 & 28.6 & 192 & 88.9 & 24 & 11.1 & 1 & & \\
\hline \multicolumn{10}{|c|}{ Tempo dos sintomas } \\
\hline 1 a 3 dias & 491 & 65.2 & 258 & 52.5 & 233 & 47.5 & 1,87 & $0,98-3,54$ & 0,0566 \\
\hline 4 a 30 dias & 216 & 28.7 & 117 & 54.2 & 99 & 45.8 & 1,75 & $0,89-3,42$ & 0,1032 \\
\hline$>30$ dias & 46 & 6.1 & 31 & 67.4 & 15 & 32.6 & 1 & & \\
\hline
\end{tabular}

*OR: odds ratio bruto; IC95\%: intervalo de confiança de $95 \%$

Na Tabela 4 são apresentados os resultados para a regressão logística múltipla. Quando o resultado de cada variável foi ajustado para as demais variáveis independentes para a classificação de risco, observou-se que os indivíduos classificados como não urgentes possuíam as seguintes características significativas: apresentavam autopercepção de que sua condição era um caso de emergência e idade igual a ou acima de 60 anos. Indivíduos com autopercepção de emergência do seu estado de saúde possuíam 9,79 mais chances de serem classificados como não urgentes (IC95\% de 6,14; 15,63). Indivíduos com idade de 60 anos ou mais apresentavam 2,66 (IC95\% de 1,$78 ; 3,97$ ) vezes mais chance de serem classificados como não urgentes.

Tabela 4. Resultados da análise de regressão logística múltipla da associação das variáveis com o risco não urgente - Piracicaba-SP, 2016.

\begin{tabular}{|c|c|c|c|c|c|}
\hline \multirow{2}{*}{ Variáveis } & \multicolumn{2}{|c|}{ Não urgente } & \multirow{2}{*}{$\mathbf{O R}^{*}$} & \multirow{2}{*}{ IC95\% } & \multirow{2}{*}{ p-valor } \\
\hline & $\mathbf{n}$ & $\%$ & & & \\
\hline \multicolumn{6}{|l|}{ Bloco proximal } \\
\hline \multicolumn{6}{|c|}{ de percepção de emergência } \\
\hline Sim & 323 & 59.8 & 9,79 & $6,14-15,63$ & $<0,0001$ \\
\hline Não & 24 & 11.1 & 1 & & \\
\hline \multicolumn{6}{|l|}{ Bloco distal } \\
\hline \multicolumn{6}{|l|}{ Idade } \\
\hline 18 a 44 anos & 122 & 32.6 & 1 & & \\
\hline 45 a 59 anos & 95 & 50.8 & 1,43 & $0,97-2,12$ & 0,4877 \\
\hline 60 anos ou mais & 129 & 66.7 & 2,66 & $1,78-3,97$ & $<0,0001$ \\
\hline
\end{tabular}

*OR: odds ratio ajustado; IC95\%: intervalo de confiança de 95\%. Nenhuma variável do bloco mesial permaneceu no modelo 


\section{DISCUSSÃO}

A análise do modelo multivariado e hierarquizado contendo variáveis, contextuais, do serviço e individuais para compreender o uso inapropriado dos serviços de UPAs evidenciou que as variáveis do bloco proximal (percepção de emergência) e do bloco distal (idade) permaneceram estatisticamente associadas ao desfecho no modelo final de regressão logística. Ao nosso conhecimento, este é um dos poucos estudos que investigaram associações com esse desfecho em usuários desses serviços por meio de um modelo hierárquico e baseado em referencial teórico amplamente consolidado na literatura científica, trazendo, portanto, importantes evidências para o planejamento em saúde.

Machado et al. ${ }^{14}$ investigaram associações entre variáveis sociodemográficas, clínicas e de utilização e a inadequação dos atendimentos realizados em uma UPA de Ouro Preto (MG), em 2012, e verificaram que a utilização inadequada da UPA teve mais chance de ocorrer em dia útil e quando havia procura direta (sem encaminhamento) pelos pacientes.

Corroborando nossos achados, estudos indicam que a autopercepção do indivíduo de que seu estado de saúde necessita de cuidados pelos serviços de saúde apresenta elevada associação de sua procura pelos serviços ${ }^{15-17}$. Para decidir sobre onde obter cuidados de saúde, os pacientes se baseiam em aspectos subjetivos das suas próprias experiências, as quais refletem situações pontuais. Desta forma, a percepção das necessidades dos pacientes é fundamental para entender a utilização dos serviços de emergência.

Ademais, observou-se diferença estatisticamente significativa para o uso de serviços de emergência entre os grupos etários pesquisados, fato também observado em diversos estudos que investigaram associações entre a idade e o uso inapropriado dos serviços de urgência ${ }^{18-24}$. Atualmente, o envelhecimento tem se tornado uma preocupação, em razão das limitações fisiológicas da senescência, da alta prevalência das doenças crônicas não transmissíveis e de inúmeras outras enfermidades que acarretam declínios físicos ou psíquicos, tornando o idoso cada vez mais dependente de cuidados, inclusive os de emergência ${ }^{22-24}$.

Verificou-se que mais da metade dos usuários que procuraram os serviços das UPAs foi classificada como apresentando necessidades pouco e/ou não urgentes, portanto estava contribuindo para a superlotação desses serviços. Esse fato também foi observado por Machado et al. ${ }^{14}$ em um estudo realizado em uma UPA de Ouro Preto (MG), no qual 58,9\% dos atendimentos investigados foram considerados "inadequados", segundo o Protocolo de Adequação de Urgências Hospitalar (PAUH). O sistema de saúde brasileiro é organizado pelas redes de atenção, com pontos de assistência de diferentes complexidades, seja primário, secundário e terciário. Por determinação, o atendimento às urgências precisa estar amparado em todos esses níveis de complexidade, cada uma dentro das suas limitações de resolutividade, inclusive na atenção primária à saúde ${ }^{1,4}$. Entretanto, segundo uma análise acerca da implantação e do desempenho das UPAs em todo o país, verificou-se que $36,7 \%$ dos usuários atendidos foram classificados como de perfil de baixo risco (azul). Os diagnósticos mais frequentes nesses casos constituíram-se principalmente de condições sensíveis à atenção primária ${ }^{25}$.

Com base nos achados do presente estudo, sugere-se a implementação de ações como a melhoria do vínculo das UPAs com os serviços de atenção básica via um sistema de referência e contrarreferência que reforce a integralidade do cuidado à saúde. Destaca-se, ainda, a necessidade de investimentos relacionados ao acesso, ao uso e à compreensão das informações e serviços de saúde, possibilitando um fluxo seguro e eficaz da população dentro da rede assistencial à saúde, ou seja, seus níveis de letramento em saúde ${ }^{26}$.Também, o fomento da contrarreferência de forma responsável à atenção básica pelos serviços de urgência ao encaminharem os casos mais sensíveis, a fim de que seja garantida a continuidade do cuidado em saúde ${ }^{27}$.

Entre as limitações do estudo, destaca-se a coleta de dados ter sido realizada em apenas duas UPAs, fato que pode ter influenciado as características das demandas apresentadas pelos usuários desses serviços. Sugerem-se novos estudos que contemplem outras abordagens metodológicas para captar informações que possam ampliar os conhecimentos sobre os fatores moduladores de demandas não urgentes desse tipo de serviço. 
Variáveis individuais como a autopercepção de caso de urgência e idade igual ou superior a 60 anos estiveram associadas ao uso não urgente dos serviços oferecidos pelas UPAs. Estudos futuros de cunho qualitativo poderão auxiliar a compreender os motivos do uso inadequado desses serviços por tais indivíduos.

\section{REFERÊNCIAS}

1. Brasil. Ministério da Saúde. Secretaria de Atenção à Saúde. Departamento de Atenção Especializada. Manual instrutivo da Rede de Atenção às Urgências e Emergências no Sistema Único de Saúde (SUS). Brasília: Editora do Ministério da Saúde; 2013.

2. Leite IC, Valente JG, Schramm JM, Daumas RP, Rodrigues RN, Santos MF, et al. Burden of disease in Brazil and its regions, 2008. Cad Saude Publica. 2015;31(7):1551-64. http://dx.doi.org/10.1590/0102-311X00111614. PMid:26248109.

3. Oliveira SN, Ramos BJ, Piazza M, Prado ML, Reibnitz KS, Souza AC. Unidade de Pronto Atendimento - UPA 24h: percepção da enfermagem. Texto Contexto Enferm. 2015;24(1):238-44. http://dx.doi. org/10.1590/0104-07072015003390011.

4. O'Dwyer G, Konder MT, Reciputti LP, Lopes MGM, Agostinho DF, Alves GF. O processo de implantação das unidades de pronto atendimento no Brasil. Rev Saude Publica. 2017;51:1-12. http://dx.doi.org/10.11606/ S1518-8787.2017051000072. PMid:29236876.

5. Morley C, Unwin M, Peterson GM, Stankovich J, Kinsman L. Emergency department crowding: A systematic review of causes, consequences and solutions. PLoS One. 2018;13(8):1-42. http://dx.doi.org/10.1371/ journal.pone.0203316

6. Tsai MH, Xirasagar S, Carroll S, Bryan CS, Gallagher PJ, Davis K, et al. Reducing high-users' visits to the emergency department by a primary care intervention for the uninsured: A retrospective study. Inquiry. 2018;55:1-12. http://dx.doi.org/10.1177/0046958018763917. PMid:29591539.

7. Cremonesi P, di Bella E, Montefiori M, Persico L. The Robustness and effectiveness of the triage system at times of overcrowding and the extra costs due to inappropriate use of emergency departments. Appl Health Econ Health Policy. 2015;13(5):507-14. http://dx.doi.org/10.1007/s40258-015-0166-5. PMid:25854901.

8. Carret MLV, Fassa AG, Paniz VMV, Soares PC. Características da demanda do serviço de saúde de emergência no Sul do Brasil. Cien Saude Colet. 2011;16(1 Suppl 1):1069-79. http://dx.doi.org/10.1590/ S1413-81232011000700039. PMid:21503455.

9. Costa JSM. Serviços de urgência e emergência hospitalar: atendimento não urgente nas redes de atenção às urgências, num contexto de transformações demográficas [tese]. Belo Horizonte: Universidade Federal de Minas Gerais; 2011.

10. Instituto Brasileiro de Geografia e Estatística. Cidades [Internet]. 2018 [citado em 2019 jan 25]. Disponível em: https://cidades.ibge.gov.br/brasil/sp/piracicaba/panorama

11. Mackway-Jones K, Marsden J, Windle J. Sistema Manchester de classificação de risco. Belo Horizonte: Grupo Brasileiro de Classificação de Risco; 2010.

12. Gomide MFS, Pinto IC, Gomide DMP, Zacharias FCM. Perfil de usuários em um serviço de pronto atendimento. Medicina. 2012;45(1):31-8.

13. Andersen RM. Revisiting the behavioral model and access to medical care: does it matter? J Health Soc Behav. 1995;36(1):1-10. http://dx.doi.org/10.2307/2137284. PMid:7738325.

14. Machado GVC, Oliveira FLP, Barbosa HAL, Giatti L, Bonolo PF. Fatores associados à utilização de um serviço de urgência/emergência, Ouro Preto, 2012. Cad Saude Colet. 2015;23(4):416-24. http://dx.doi. org/10.1590/1414-462X201500040177.

15. Abreu KP, Pelegrini AHW, Marques GQ, Lima MADS. Percepções de urgência para usuários e motivos de utilização do serviço de atendimento pré-hospitalar móvel. Rev Gaúcha Enferm. 2012;33(2):146-52. http:// dx.doi.org/10.1590/S1983-14472012000200021. PMid:23155593.

16. Coster JE, Turner JK, Bradbury D, Cantrell A. Why do people choose emergency and urgent care services? A rapid review utilizing a systematic literature search and narrative synthesis. Acad Emerg Med. 2017;24(9):1137-49. http://dx.doi.org/10.1111/acem.13220. PMid:28493626.

17. Burns TR. Contributing factors of frequent use of the emergency department: A synthesis. Int Emerg Nurs. 2017;35:51-5. http://dx.doi.org/10.1016/j.ienj.2017.06.001. PMid:28676296. 
18. Andrews $\mathrm{H}$, Kass L. Non-urgent use of emergency departments: populations most likely to overestimate illness severity. Intern Emerg Med. 2018;13(6):893-900. http://dx.doi.org/10.1007/s11739-018-1792-3. PMid:29380133.

19. Veras RP, Oliveira M. Envelhecer no Brasil: a construção de um modelo de cuidado. Cien Saude Colet. 2018;23(6):1929-36. http://dx.doi.org/10.1590/1413-81232018236.04722018. PMid:29972500.

20. Gomide MFS, Pinto IC, Gomide DMP, Zacharias FCM. Perfil de usuários em um serviço de pronto atendimento. Medicina. 2012;45(1):31-8.

21. Acosta AM, Lima MADS. Frequent users of emergency services: associated factors and reasons for seeking care. Rev Latino-Am Enfermagem. 2015;23(2):337-44. http://dx.doi.org/10.1590/0104-1169.0072.2560. PMid:26039306.

22. Chiavegatto Filho ADP, Wang YP, Malik AM, Takaoka J, Viana MC, Andrade LH. Determinantes do uso de serviços de saúde: análise multinível da Região Metropolitana de São Paulo. Rev Saude Publica. 2015;49:111. http://dx.doi.org/10.1590/S0034-8910.2015049005246. PMid:25741652.

23. Gruneir A, Silver MJ, Rochon PA. Emergency department use by older adults: A literature review on trends, appropriateness, and consequences of unmet health care needs. Med Care Res Rev. 2011;68(2):131-55. http://dx.doi.org/10.1177/1077558710379422. PMid:20829235.

24. Faulkner D, Law J.The'unnecessary'use of emergency departments by older people: findings from hospital data, hospital staff and older people. Aust Health Rev. 2015;39(5):544-51. http://dx.doi.org/10.1071/ AH14185. PMid:25913422.

25. Brasil. Conselho Nacional de Secretários de Saúde. Rede de Atenção às Urgências e Emergências: Avaliação da implantação e do desempenho das Unidades de Pronto Atendimento (UPAs). Brasília: CONASS; 2015.

26. Mialhe FL, Moraes KL, Brasil VV, Sampaio HAC. Letramento em saúde e promoção da saúde. In: Pelicioni MCF, Mialhe FL. Educação e Promoção da Saúde: Teoria e prática. São Paulo: Santos; 2019.

27. Stein AT, Harzheim E, Costa M, Busnello E, Rodrigues LC. The relevance of continuity of care: a solution for the chaos in the emergency services. Fam Pract. 2002;19(2):207-10. http://dx.doi.org/10.1093/ fampra/19.2.207. PMid:11906990. 